\title{
Design of Sequencing Medium Access Control to Improve the Performance of Wireless Networks
}

\author{
P. Venkata Krishna and N. Ch. S. N. Iyengar \\ School of Computing Sciences, VIT University, Vellore, India
}

\begin{abstract}
This paper presents a technique for accessing channel based on sequencing technique for 802.11 wireless networks. The objective of this method is to reduce, as well as to avoid the number of collisions while accessing channel by many nodes at the same time. A concept of sequence number is introduced to avoid collisions. The transmission of a node takes place after checking the sequence number. MAC layer issues are very important while accessing channel over wireless networks. Simulation results show that the performance of sequence MAC is improved significantly when compared with legacy MAC.
\end{abstract}

Keywords: QoS, 802.11 MAC, DCF, sequencing etc.

\section{Introduction}

In wireless network, multiple stations contend for access to the shared channel. The cost of collision detection in wireless networks is much higher than in wired networks since stations cannot detect the collision without explicit feedback from the receiver. For this reason, more efficient contention resolution algorithms are desired for wireless networks to reduce the collision probability among various contending stations. In wireless networks, a station usually can only learn about a collision when the transmission is finished and the expected acknowledgment does not come back. Consequently, a collision will cause for last of the entire packet transmission duration resulting in higher collision cost in wireless networks compared with wired networks.

In wireless networks, the MAC protocol is the main element that determines the efficiency of sharing the limited communication bandwidth of the wireless channel. The fraction of channel bandwidth used by successfully transmitted messages gives a good indication of the protocol efficiency, and its maximum value is referred to as protocol capacity. If a station has an exact knowledge of the network status, it is possible to tune its backoff algorithm to achieve a protocol capacity very close to its theoretical bound. But it is very difficult to estimate the load conditions of the network and hence effective media access protocols are required to minimize the number of collisions. Another major design goal of media access protocols is to avoid communication overhead. Development of efficient MAC protocols providing both high throughput performance for data traffic and good quality of service support for real-time traffic is the current major focus in distributed contention-based MAC protocol research. This paper studies the performance of the IEEE 802.11 DCF-based Media Access Control protocol and proposes a Sequencing Medium Access Control protocol based on the concept of grouping the time slots of nodes in the network. In DCF-based multiple access control protocols, two categories of overhead are usually associated with contention resolution. One is channel idle overhead, where all contending stations are waiting to transmit. Another is collision overhead, which occurs when multiple contending stations attempt to transmit simultaneously. Either idle overhead or collision overhead being large, contention resolution algorithm would be inefficient. The proposed design based on sequencing technique tries to minimize both the idle and the collision 
overheads by minimizing the backoff procedure intervals among the nodes.

This paper investigates the robustness of the sequencing method and presents a study on its performance. The next section of the paper describes preliminaries of wireless MAC and its issues. The overview of existing approach is discussed in Section 3. Section 4 presents' related works and Section 5 describes sequencing MAC and its performance issues. Finally, Section 6 gives results and discussions on the sequencing technique followed by conclusions.

\section{Preliminaries}

Generally, MAC protocols are classified into two types i.e., centralized (reservation-based) and distributed (contention-based) protocols. Reservation-based protocols are used in access point-based networks (single hop networks). The access point uses polling concept before assigning channel to a node. These reservation protocols have to provide quality of service (QoS) guarantees for real-time traffic. In Centralized protocols, central controller is responsible for accessing wireless channel. 802.11e uses PCF (Point Coordination Function) for Centralized protocols. Contention-based protocols are distributed algorithms that allow nodes in an ad hoc network (multi hop network) to be able to communicate effectively over the shared channel and are largely based on Carrier Sense Multiple Access with Collision Avoidance (CSMA/CA). ALOHA, HIPERLAN, MACAW, and IEEE 802.11 Distributed Function (DCF) are just some of the many contentionbased protocols that have been proposed. In general, contention-based protocols are useful to increase channel capacity as reservationbased protocols underutilize the channel. Furthermore, contention-based protocols will be more able to meet dynamic requirements and demands of a wireless network along with QoS guarantees. The distributed protocol does not require any co-ordination for wireless channel access. CSMA protocol is the known method of channel access for distributed protocols. The IEEE 802.11 uses DCF (Distributed Coordination Function) for distributed protocols [1] [2] [4] [6][7].
The IEEE 802.11 DCF supports two basic access methods i.e., accessing the channels with and without RTS (Request To Send)/CTS (Clear To Send) handshake. The use of RTS/CTS handshake is avoiding the problems of hidden nodes and exposed nodes.

Hidden node: If a node is located within the transmission range of the receiver, but not of the sender, it will not hear the packet exchange and may start sending the packet to the same receiver. This results in costly data packet collisions. Such a node is called a hidden node. In a wireless network, every node has a signal receiving range and a carrier sensing range. A node cannot receive any packet from beyond its receiving range and cannot sense any neighboring transmission beyond the carrier sensing range. Assuming that these two ranges are equal, we can have a scenario where nodes are hidden from each other i.e. outside the range and cause costly data packet collisions.

Exposed node problem arises when the sensing mechanism prevents parallel transmission, from two or more terminals, toward receivers that would not observe collision, as the receivers are located far apart.

Inter Frame Space: The 802.11 DCF protocol uses various time intervals between frames called Inter Frame Spaces (IFS). The 802.11

\begin{tabular}{|l|l|}
\hline IFS Name & Description \\
\hline \hline SIFS & $\begin{array}{l}\text { Short Inter Frame Space (SIFS) is used to } \\
\text { separate transmissions belonging to the } \\
\text { single dialog (Fragment-ACK) and it is } \\
\text { the minimum inter frame space. This } \\
\text { value for 802.11 PHY is fixed to 28 ms. }\end{array}$ \\
\hline PIFS & $\begin{array}{l}\text { Point Coordination IFS (PIFS) is used } \\
\text { by the Access Point (or Point Coordinator) } \\
\text { to gain access over the medium before } \\
\text { any other station. The value is } \\
\text { SIFS + Slot Time, i.e. 78 ms (50+28). }\end{array}$ \\
\hline DIFS & $\begin{array}{l}\text { Distributed IFS (DIFS) is the inter frame } \\
\text { space used for a station willing to start } \\
\text { a new transmission, which is calculated } \\
\text { as PIFS plus one slot time, i.e. 128 ms } \\
\text { (50+78). }\end{array}$ \\
\hline EIFS & $\begin{array}{l}\text { Extended IFS (EIFS) is a longer IFS } \\
\text { used by a station that has received a } \\
\text { packet it could not understand. This is } \\
\text { needed to prevent the station from } \\
\text { colliding with a future packet belonging } \\
\text { to the current dialog. Normally, this } \\
\text { larger than DIFS. }\end{array}$ \\
\hline
\end{tabular}

Table 1. IFS types. 
DCF protocol uses the IFS to determine the medium is idle through the use of the carriersense function for the interval specified. Four different IFSs are defined to provide priority levels for access to the wireless media and they are listed in the above Table 1.

\section{Overveiw of the 802.11 DCF Protocol}

The IEEE 802.11 MAC layer protocol provides asynchronous, time-bounded, and contentionfree access control on top of physical layer. The basic access method in the IEEE 802.11 MAC protocol is the distributed coordination function (DCF) which is a CSMA/CA MAC protocol. In addition to the DCF, the IEEE 802.11 also incorporates an alternative access method known as the point coordination function (PCF) - an access method that is similar to a polling system and uses a point coordinator to determine which station has the right to transmit.

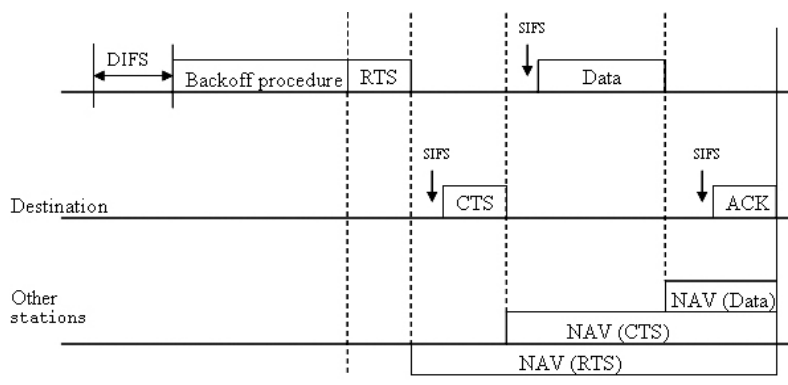

Figure 1. 802.11 DCF protocol.

In DCF protocol, as shown in Figure 1, a node senses the channel for ongoing transmissions before sending a packet. If the channel is already in use, the node waits for a free DIFS and a random backoff time before re-attempting the transmission. If another station is using the medium during the backoff time of the station, the backoff timer stops. On the other hand, if the channel is not currently in use i.e., if the channel is idle during DIFS, the node begins transmission.

This method uses only DATA frames and ACK frames. The station wishing to send a data frame first senses the channel and may proceed with transmission of a DATA frame if the medium is sensed to be idle for an interval larger than DIFS.
When the DATA frame is transmitted, all other stations hearing the frame set their network allocation vector, NAV, based on the duration field value in the data frame received. The duration field in the data frame includes the SIFS and the ACK frame transmission times following the DATA frame. By using this virtual carrier sensing mechanism, all stations that hear the DATA frame will refrain from transmitting during the ACK frame transmission, thus reducing the frame collisions. If the medium is busy, the transmitting station will wait until the end of the current transmission. It will then further wait for an additional DIFS time period, and then the station defers for a random backoff period before transmitting. Having received a DATA frame correctly, the destination station waits for a SIFS interval immediately following the reception of the DATA frame, and then transmits an ACK frame back to the source station, indicating that the DATA frame has been received correctly. As when a station becomes ready to transmit, it senses the medium for a period of DIFS. If the station senses that the medium is busy during this interval, it defers transmission to later time which depends on the backoff time. The backoff time is calculated using the following relationship.

Backoff Time $=$ Random ()$*$ aSlotTime $(3.1)$

$\operatorname{Random}()$ is a value in the interval $[0, \mathrm{CW}]$, where CW, Contention Window, takes a value between aCWmin and aCWmax i.e. aCWmin $<\mathrm{CW}<\mathrm{aCWmax}$. The values of aCWmin and aCWmax are defined for Physical layer. aSlotTime is the value of the defined by the physical layer (PHY characteristic) [6] [7].

The Distributed Coordination Function (DCF) adopts a slotted binary exponential backoff technique [4]. In this, the time immediately following an idle distributed DIFS is slotted, and the station is allowed to transmit only at the beginning of each slot time, which is equal to the time needed at any station to detect the transmission of a packet from any other station. Positive acknowledgments are employed to assure the successful reception of each packet transmission. This is accomplished by the receiver (immediately following the reception of the data 
frame) which initiates the transmission of an acknowledgment frame after a time interval, SIFS, which is less than the DIFS. If an acknowledgment is not received, the data frame is presumed to have been lost, and a retransmission will take place.

In IEEE 802.11 DCF all stations begin their contention resolution stage (i.e., random backoff stage) after the channel has become idle. Therefore, the chosen range of random backoff period is critical to the performance of the MAC protocol.

The DCF has another backoff procedure known as binary exponential backoff (BEB) [4][13] to resolve channel contention. In this, a station that wants to transmit a data packet must first carrier-sense the medium. If the channel is idle for at least DIFS duration, the node randomly picks a backoff counter value. This value is drawn from a uniform distribution over the interval $[0, \mathrm{CW}-1]$, where $\mathrm{CW}$ is the size of the node's contention window. If the medium is sensed to be busy, the node must defer its transmission until the medium is idle for at least DIFS duration before selecting a backoff value.

The backoff value represents the number of idle slots a node has to wait before it can transmit. For each idle slot it senses on the channel, the node decrements its backoff counter by one. If the channel is sensed to be busy, the node freezes its backoff counter until the channel is idle for at least the DIFS duration before it resumes decrementing the counter.

When the backoff counter reaches 0 , it transmits an RTS. If a node receives an RTS, it replies with CTS. Upon receiving the CTS, the node then transmits the data packet, which is then replied with an ACK. All the nodes that overhear either the RTS or the CTS set their Network Allocation Vector (NAV) for this duration and defer transmitting while their NAV is set. As a consequence, this allows for the collision-free transmission of the data and ACK. This mechanism of deferring transmission based on the NAV is known as virtual carrier sensing and it effectively reserves the channel. The combination of the virtual and physical carrier sensing mechanisms ensures that collisions only occur when two nodes pick the same slot to transmit. Since a node cannot sense the medium while transmitting, an absence of a reply (i.e., a CTS or an $\mathrm{ACK}$ ) is taken as an indicator that a collision has occurred. When this happens, the node doubles its contention window and reselects a new backoff value. The nodes that sense the collision will set their NAV to the Extended Inter-frame Space (EIFS) duration. This duration is defined to allow an ACK to be successfully transmitted before other transmissions recommence. As the number of collisions increases, nodes exponentially increase their contention windows so as to reduce the probability of another collision. A node starts with its $\mathrm{CW}=\mathrm{CWmin}$ and with each collision it doubles its backoff value until it reaches the maximum value of CWmax. The values of CWmin and CWmax depend on the physical layer used. Once the node is successful in its transmission attempt, it resets its $\mathrm{CW}$ back to the minimum value. The total duration of one successful packet transmission, which is the interval between the end of the last successful transmission and the reception of the ACK for the current packet, is known as one transmission cycle.

\section{Related Works}

There have been significant works carried out the recent years related to 802.11 MAC protocols. Jaehyuk et al. [1] propose DCF-based CSMA method based on early backoff announcement to increase the throughput of the network. N. Vaidya et al. [2] proposes distributed fair scheduling concepts to allocate bandwidth for wireless networks. Fitzek et al proposes a framework for CDMA systems that improves the performance. Kiyoshi et al [10] describes a backoff procedure for improving satutation throughput in 802.11 DCF protocol. Yoshifumi Nishida [11] explains performance of 802.11 DCF MAC for TCP/IP protocols. Hwangnam Kim et al [12] proposes a Model-based Frame scheduling for 802.11 WLANs. Most of the related works are explained based on the setting of backoff timer. Increase and decrease of the control window for nodes while transmitting the packets is a serious concern and improper setting of control window causes more collisions. We propose a design of MAC based on 
the Sequencing Technique which reduces the number of collisions. The basic Sequencing Technique is explained in $[14,15]$. In this paper, we present DCF-based channel access based on the Sequencing Technique. The proposed Sequencing MAC minimizes the number of collisions by restricting the nodes from transmission, if their sequence number saturates. Also, this paper presents the performance analysis of the Sequencing MAC over DCF protocol. We have done our simulations using NS-2 simulator and the results show that the performance of Sequencing MAC is good.

\section{Sequencing MAC}

The basic sequencing method sets a value called sequence number ' $\mathrm{K}$ ' to all the nodes so that each node has to limit its transmissions to less than or equal to ' $\mathrm{K}$ ' in a time slot. The sequencing number will be assigned to every node before it accesses the medium. As the number attempts are limited during a time slot, the sequencing technique reduces number collisions. The cost of implementing sequencing technique will be minimal because use of the simple counters is sufficient. The collisions are avoided by comparing the counter values of contending nodes. A node having a less counter value will be deferred from transmission. The sequencing technique is significantly reducing number of collisions when compared with legacy MAC protocols.

There are two methods exist while accessing medium access i.e., i) With RTS/CTS ii) Without RTS/CTS. The RTS/CTS mechanism is used to avoid the problems of hidden node and exposed nodes. In this, if a node wants to transmit a packet, first it sends RTS packet to the destination. There is only the need for control channel procedure before accessing the channel in the case without RTS/CTS method.

The following figure shows an example scenario of how the RTS packets are grouped for each sequence value. The numbers 1 to 11 and so on refer to the RTS packet number transmitted by that particular node.
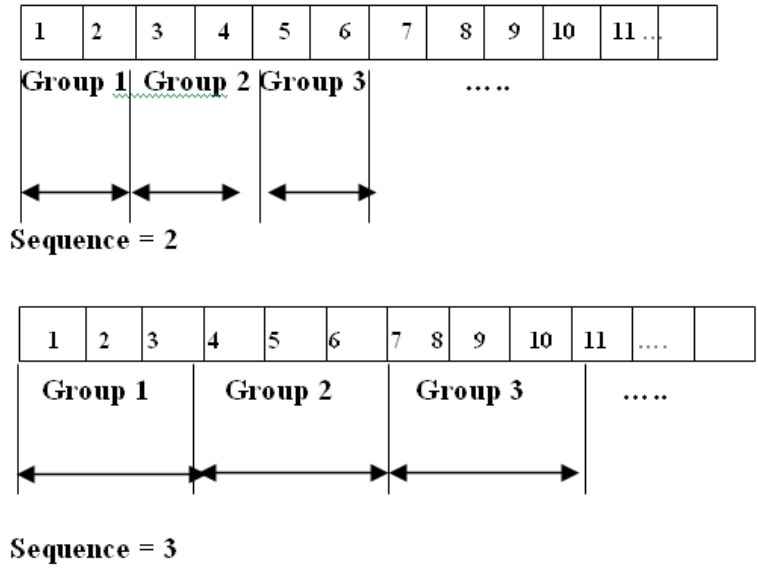

Figure 2. Example scenario for grouping of RTS.

The above discussed sequence value pairs are used to selectively drop RTS packets. The question that arises is how dropping of RTS packets, irrespective of the network conditions, results in improving the performance of the networks? The sequencing technique is used to drop packets so that we refrain from initiating a data transfer from one node to another, which may later prove to be costly if a data packet collision occurs due to any of the problems elucidated in previous sections.

When a Data/Acknowledgement packet collision occurs, the whole contention resolution phase followed by the RTS/CTS exchange is repeated. This leads to loss of time and the net throughput low.

Dropping RTS avoids such situations and the concept of sequencing is implemented for the above-mentioned reasons. With increasing value of sequence and number of nodes, lesser number of RTS packets are dropped thereby making the system coverage a steady state.

Figure 3 shows the timeline analysis of sequencing technique. Here, three nodes are accessing medium to send information. The sequence number is set to two and a counter is maintained at every node to count the number of attempts by node. A node drops packets by comparing the sequence number and counter value. A node is deferred from transmission if the counter value reaches equal to sequence number. A node can start packet transmission after resetting the counters to zero. Normally, Counters will be refreshed for every timeslot. 


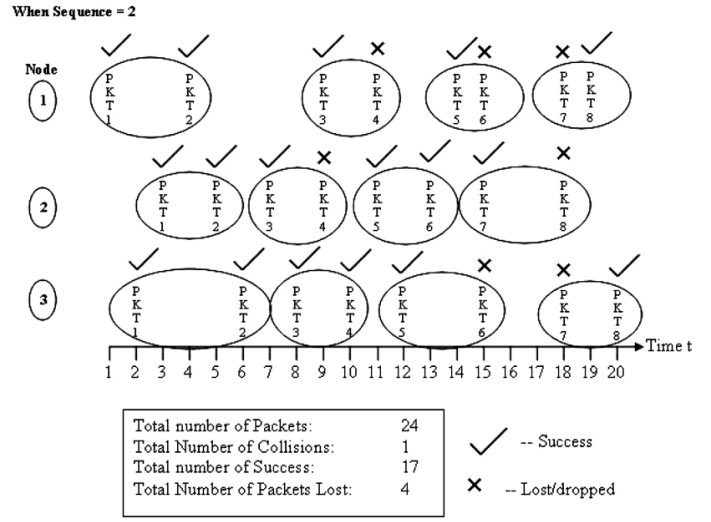

Figure 3. Timeline Analysis.

It is observed from Figure 3 that the total number of transmissions from three nodes is 24 and number of packets successful is 17 . Also, the number of collisions is very minimal i.e., one, and the number of packets dropped is four. Reducing the number of collisions is the main advantage of the sequencing technique. Number of collisions will increase to four if sequencing technique is not used and the number of packet losses will be 11 for the above example shown in Figure 3. Figure 4 shows the flow diagram for sequencing technique.

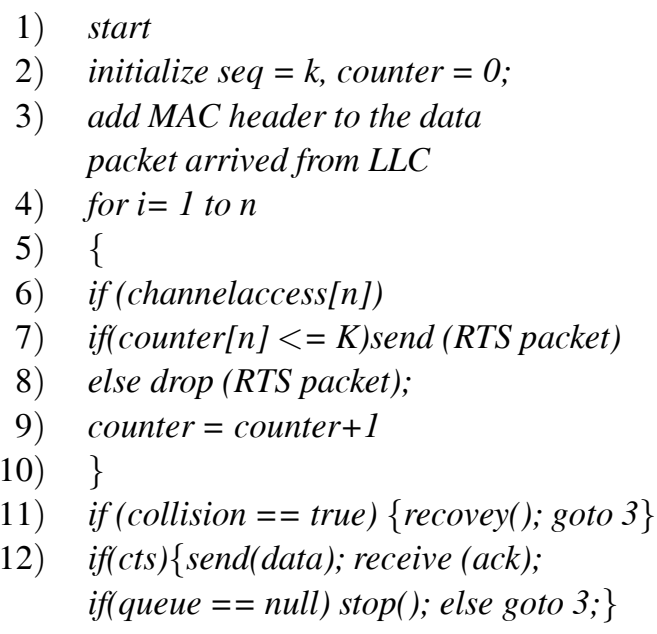

Sequencing Algorithm

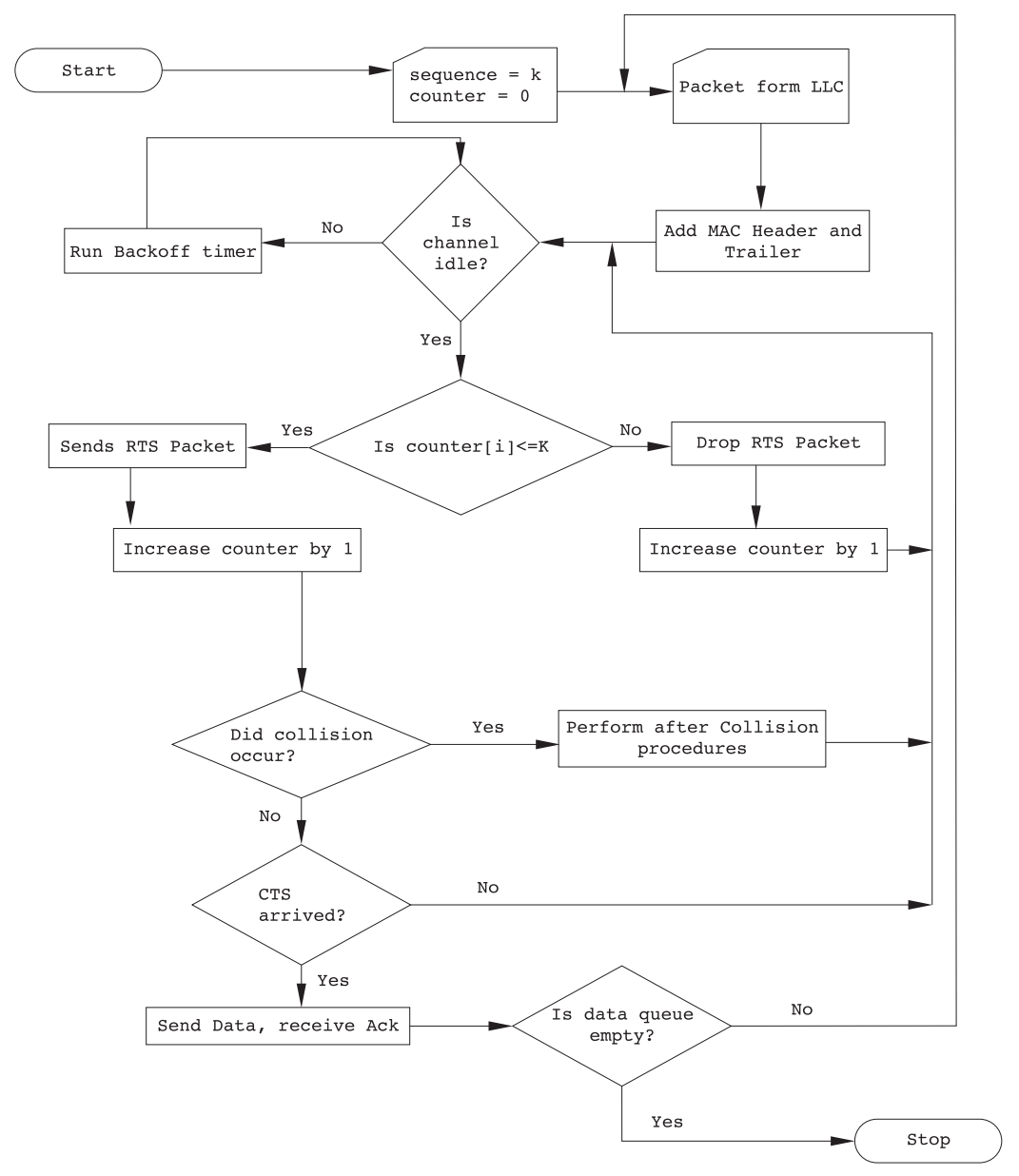

Figure 4. Flow diagram for sequence procedure. 
The simulations are performed using NS2 and the performance of the sequencing technique is discussed in the next section.

\section{Results and Discussions}

In this section we present performance of sequencing technique. The results are obtained using Network Simulator tool. The performance of sequencing technique is compared with 802.11 MAC DCF. The simulations are performed by increasing number of nodes as well as sequence number, $\mathrm{K}$.

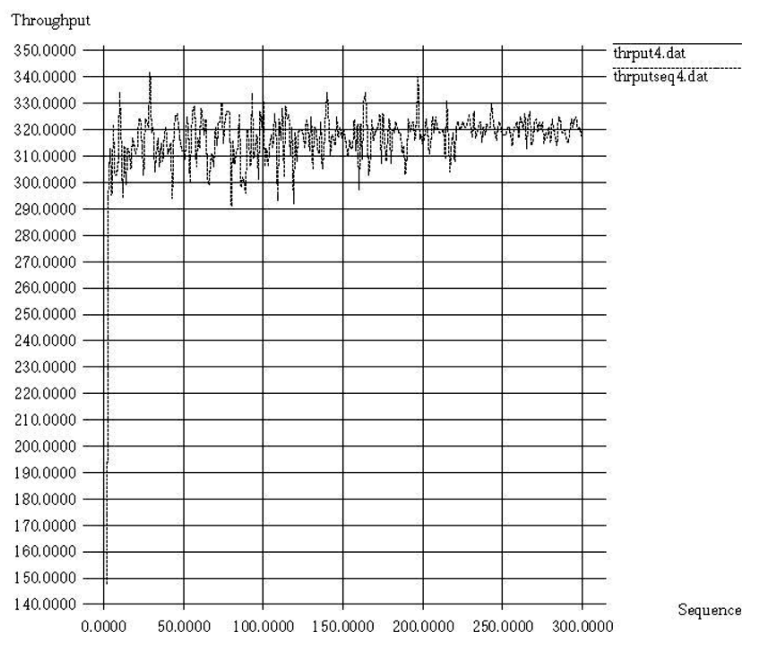

Figure 5. Comparison of throughput for MAC 802.11 and MAC 802.11Seq for Four Nodes.

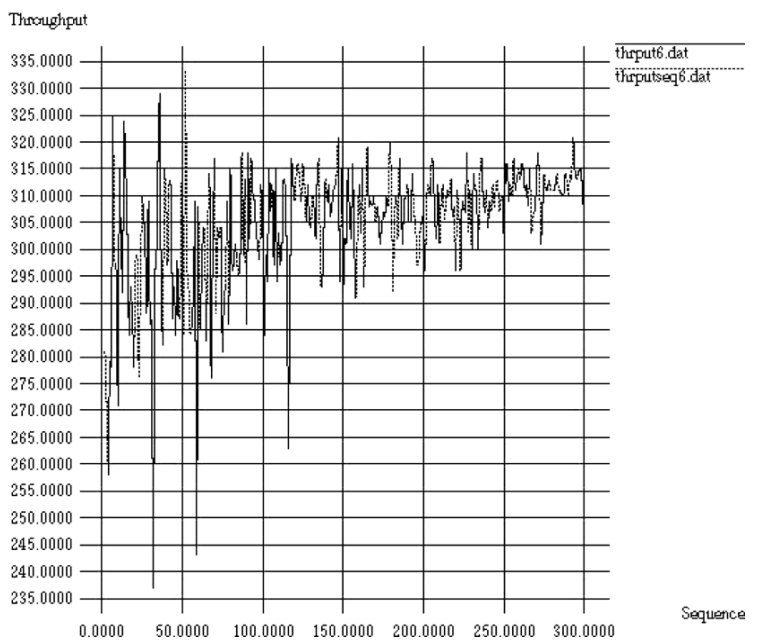

Figure 6. Comparison of throughput for MAC 802.11 and MAC 802.11Seq for Six Nodes.

\subsection{Four Node Scenario}

Let us now consider the 4-node scenario shown in Figure 5. The performance analysis is done by taking different values of sequence numbers and the throughput of the system is estimated for MAC-802.11Seq with the throughput for MAC802.11 .

The observation from Figure 5 is that the throughput for MAC-802.11Seq crosses the throughput line for MAC-802.11 at many places. The throughput for MAC-802.11Seq is 341 at the sequence value 34 . The throughput for MAC802.11 is 320 .

\subsection{Six Node Scenario}

Let us now consider the 6-node scenario shown in Figure 6. The performance is shown by obtaining the throughput for MAC-802.11Seq with the throughput for MAC-802.11 while increasing sequence numbers.

It is observed from Figure 6 that the throughput for MAC-802.11Seq is 333 at the sequence value 51. The throughput for MAC-802.11 is 300 .

Finally, Figure 7 shows overall throughput comparison of $802.11 \mathrm{seq}$ and 802.11 at various nodes. It is observed that the throughput of the sequencing technique is improved significantly when compared with legacy system. Our

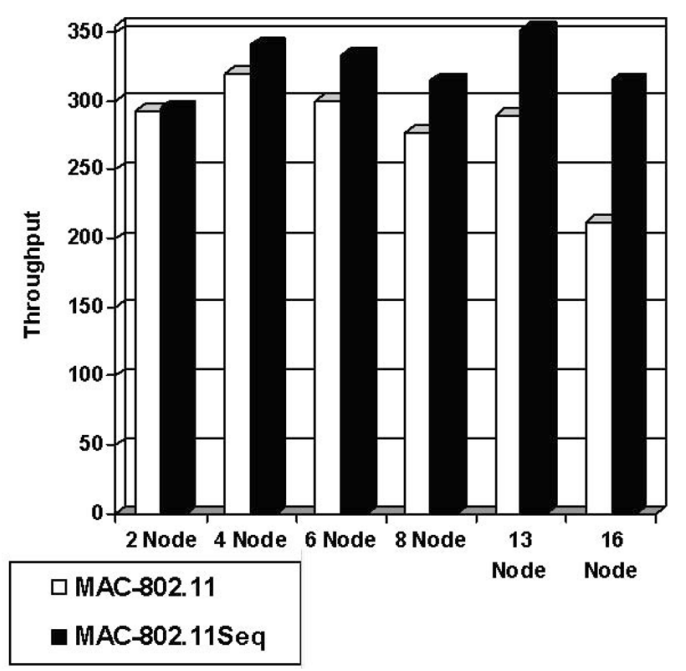

Figure 7. Throughput comparison for Different Nodes. 


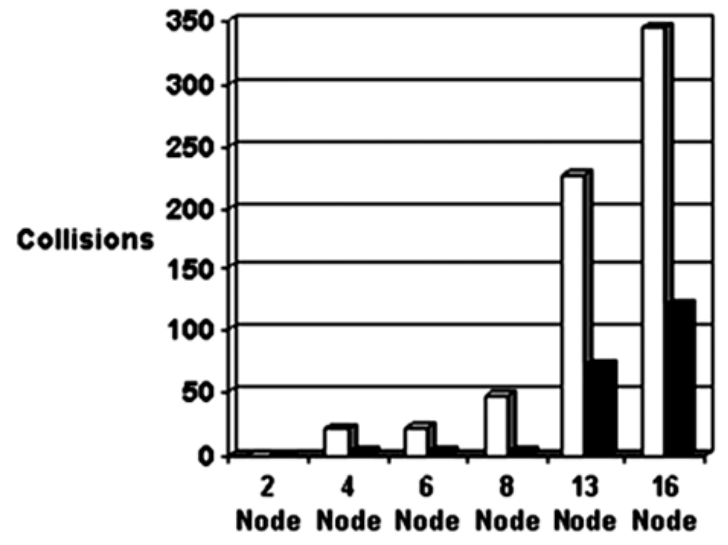

口MAC-802.11Collision

- MAC-802.11SeqCollision

Figure 8. Comparison of collisions for different numbers of Nodes.

experiments show that the performance of sequencing technique is increased to $79 \%$ when compared with legacy systems. And there is $50 \%$ minimum decrease in collisions.

Our simulation proves that the number of collisions can be reduced using MAC 802.11 seq method and the performance graph is shown in the following Figure 8.

\section{Conclusion and Future Work}

A sequencing medium access method is presented in this paper. It is observed that a design of sequencing medium access protocol reduces the number of collisions and increases overall throughput of the network. The performance of the sequencing technique depends on the sequence number as it is observed that throughout the system it improves when there is a increase in sequence number. Also, collisions are reduced when sequence number is increased. Optimal value of sequence number depends on network conditions. A study of Sequencing MAC on other wireless networks like sensor networks will be the scope for future work.

\section{References}

[1] J. CHOI, J. Yoo, S. ChoI, C. KIM, EBA: An Enhancement of the IEEE 802.11 DCF via Distributed Reservation. IEEE Trans. On Mobile Computing, 4(4) (July/August 2005), pp. 378-390.
[2] N. VAidya, S. GuPTA, P. BAHL, Distributed Fair Scheduling in Wireless LAN. IEEE Trans. On Mobile Computing, 4(6) (November/December 2005), pp. 616-628.

[3] F. FitzeK, A. Kopsel, A. Wolisz, M. Krishnam, M. REISSLEIN, Providing Application-Level QoS in $3 \mathrm{G} / 4 \mathrm{G}$ Wireless Systems: A Comprehensive Framework Based on Multirate CDMA. IEEE Wireless Communications, (April 2002).

[4] W. Pattara-Atikom, P. K. Murthy, S. BanarJEE, Distributed Mechanisms for Quality of Service (QoS) in Wireless LANs. IEEE Wireless Communications, (June 2003).

[5] V. Marques, R. L. Aguiar, C. Garcia, J. I. Moreno, C. Beaujeam, E. Melin, M. Liebsch, An IP-based QoS Architecture for 4G Operator Scenarios. IEEE Wireless Communications, (June 2003).

[6] S. Mangold, S. Choi, G. R. Hiertz, O. Klein, B. WALKE, Analysis of IEEE 802.11E for QoS Support in Wireless LANs. IEEE Wireless Communications, (December 2003).

[7] H. Zhu, M. Li, I. Chlamtac, B. Prabhakaran, A Survey of Quality of Service in IEEE 802.11 Networks. IEEE Wireless Communications, (August 2004), pp. 6-14.

[8] J. SCHILlER, Mobile Communications. Pearson Education Limited, 2001.

[9] NS2 SIMULATOR, http://www.isi.edu/nsnam/ns/tutorial/ ns.html

[10] K. TAKAHASHI, T. TsubOI, A Backoff Algorithm for Improving Saturation Throughput in IEEE 802.11 DCF.

http://www . eurasip.org/Proceedings/Ext/ IST05/papers/368.pdf

[11] Y. NISHIDA, Enhancing 802.11 DCF MAC for TCP/IP Communication. Proc. of WCNC 2005, IEEE Communications Society, (2005), pp. 16201625.

[12] H. KIM, J. C. HoU, Improving Protocol Capacity with Model-based Frame Scheduling in IEEE 802.11-operated WLANs. Proc. of MobiCom 2003, ACM, (September 2003), pp. 190-204.

[13] P. KARN, MACA - A New channel access method for packet radio. Proc. of 9th ARRL Computer Networking conference, (1990), pp. 134-140.

[14] P. V. Krishna, N. CH. S. N. IYengar, Sequencing Technique: an enhancement to 802.11 medium access control to improve the performance of wireless networks. Int. J on Computer Networks and Distributed Systems, (in press), (2007).

[15] P. V. KRISHNA, A Study of Quality of Service Metrics for Wireless Systems. Ph.D thesis, VIT University, January 2008. 
Received: February, 2007

Accepted: November,2007

Contact addresses:

P. Venkata Krishna

School of Computing Sciences

VIT University

Vellore

TN

India

e-mail: parimalavk@gmail.com

N. Ch. S. N. Iyengar

School of Computing Sciences

VIT University

Vellore

India

P. VENKATA KRISHNA is a Senior Lecturer at School of Computing Sciences, VIT University, Vellore, India. He received his B. Tech in Electronics and Communication Engineering from Sri Venkateswara University, Tirupathi, India, M. Tech in Computer Science \& Engineering from REC, Calicut, India and he is doing his Ph.D. at VIT University, Vellore, India. His research interests include mobile and wireless systems and grid computing. He has published several papers in national and international journals. He was the editor of 2003, 2004 and 2006 ObCom International conference proceedings and he is a founding member of ObCom International Conference. He has authored several books on Computer Networks and Programming Languages. He is the reviewer for many reputed journals like IET Journal on Communications, International Journal of Security and Networks (IJSN) etc.

DR. N. CH. S. N. IYENGAR is a Professor at the School of Computing Sciences at VIT University, Vellore India. He received his M.Sc (Applied Mathematics) from Kakatiya University, Andhra Pradesh, India, M.E (Computer Science and Engineering) from Anna University, Chennai, India \& Ph.D. from Regional Engineering College Warangal (presently known as NIT Warangal). His research interests include Fluid Dynamics (Porus Media), information security models, Ebusiness applications, agent technologies; QoS in networks; ontologybased Web technologies and cryptography. He has authored more than 30 textbooks for various subjects like applied mathematics, discrete mathematics, data structures and algorithms etc. He has authored more than 70 papers in national and International conferences and journals. $\mathrm{He}$ has been serving for various International and national conferences in various capacities. $\mathrm{He}$ is the editor of the proceedings of MobiComNet 2004 International conference and the founding member of MobiComNet Conferences. 
